\title{
Une démarche pour anticiper le déploiement d'un PLM dans une entreprise « libérée » - Cas du groupe Poult
}

\section{An approach to anticipate PLM solution deployment in a freedom form company - the real case study of Group Poult}

\author{
Laureline $\mathrm{Plo}^{1,2}$, Vincent Robin ${ }^{2}$, Philippe Girard ${ }^{2}$ \\ ${ }^{1}$ Groupe POULT - Site de Montauban - 1500 Chemin Du Quart, BP133, 82001 Montauban - France, \\ I.plo@groupe-poult.fr \\ ${ }^{2}$ Laboratoire IMS CNRS UMR 5218 - Université de Bordeaux, 351 cours de la libération, 33405 Talence - France, \\ vincent.robin@u-bordeaux.fr , philippe.girard@ims-bordeaux.fr
}

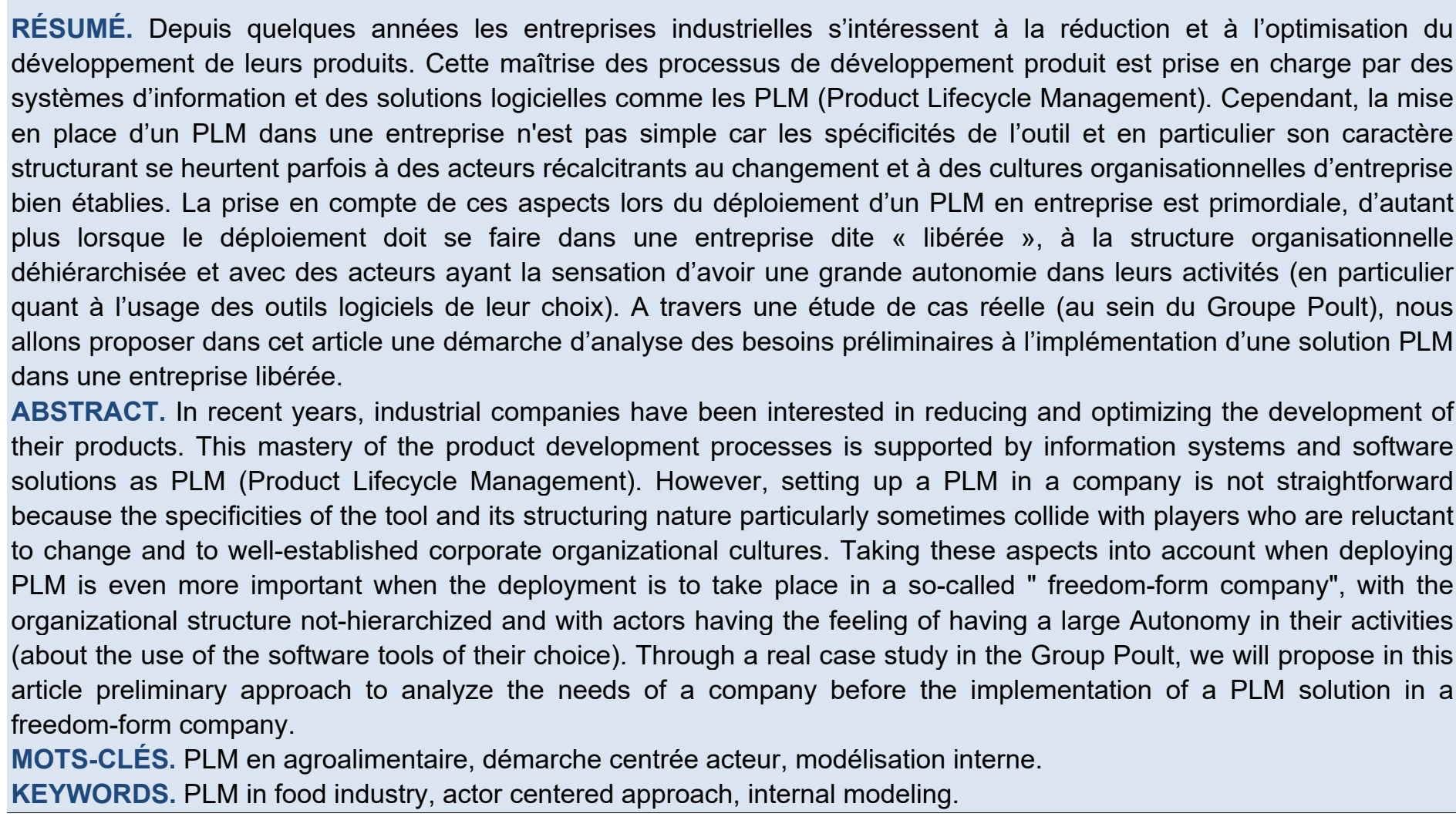

\section{Introduction}

Dans le contexte économique actuel qui peut parfois paraître comme incertain pour certaines entreprises, nombre d'entre-elles cherchent à recentrer leur activité sur leur « métier originel/propre », leur «savoir-faire" tout en promouvant des stratégies fondées sur l'innovation. Ces stratégies conduisent le plus souvent à des « innovations » sur les produits et les processus des entreprises mais aussi parfois (mais plus rarement) sur leur organisation dans le but d'accroitre leur compétitivité et leurs performances. L'une des évolutions organisationnelles perçue comme une innovation concerne la création de réseaux collaboratifs et participatifs internes aux entreprises dans le cadre de la mise en place de modes de management dynamiques favorisant la créativité et l'épanouissement des acteurs. L'objectif est « de faciliter la mise en place de structures plus centrées sur les équipes, les projets et les groupes de travail à responsabilité élargie afin d'optimiser la conduite de projets innovants » [ZAG 07]. Ce type d'organisation est souvent décrit comme l'entreprise " libérée ». Cette modalité d'organisation, bâtie sur la base de méthode de "dé-hiérarchisation massive », revient à rendre 
l'entreprise théoriquement plus performante avec des employés libérés de la hiérarchie et du contrôle. Ces changements que nous pouvons résumer par «moins d'échelons hiérarchiques pour encourager la créativité » ont été mis en place par plusieurs entreprises mais selon différents niveaux : W.L Gore \& Associates, Harley Davidson, Morning Star aux Etats-Unis, Favi, Chroni Flex ou encore le Groupe Poult en France [PET 12], [WAN 12], [CAV 15].

Pour accompagner la mise en place et le fonctionnement de telles organisations, les entreprises mènent aussi des réflexions quant à la structuration de leur système d'information (SI) et des évolutions du SI ont souvent lieu. Celles-ci induisent « des changements dans l'organisation du travail, en utilisant de nouveaux modes de collaboration, coopération et communication entre les acteurs » [ZAG 07]. Pour dynamiser la collaboration, les SI intègrent depuis une quinzaine d'années de nouvelles solutions logicielles telles que les espaces de travail partagés, les réseaux sociaux et des outils métiers qui tendent eux-aussi à offrir des interfaces et des espaces favorisant les collaborations (ERP, PLM, etc.) [HER 05]. Ces solutions, supports aux processus (de conception notamment) sont perçues comme pouvant apporter une plus-value aux pratiques de développement d'un produit/projet et optimiser les processus métier de l'entreprise [LEL 11], [KUN 15]. Besson et Rowe [BES 07] ont identifié les technologies de l'information comme l'une des forces majeures du changement mais peu de travaux se sont focalisés sur les changements que le PLM pouvait générer dans une organisation de type « entreprise libérée ».

Notre question de recherche est donc de savoir comment il est possible de prendre en compte la complexité de l'organisation et des processus collaboratifs d'innovation d'une "entreprise libérée » lors de la mise en place d'une solution de type PLM pour faire en sorte que son déploiement soit vecteur d'amélioration des performances du groupe et des pratiques professionnelles de ses acteurs. Dans cet article nous ne présenterons la démarche que nous avons définie puis mise en œuvre dans le cadre d'une thèse CIFRE entre le Groupe Poult et des membres du laboratoire IMS de l'Université de Bordeaux pour répondre à notre question de recherche. La première section de l'article décrira le processus de déploiement "classique» d'un PLM dans une entreprise et nous présenterons notre proposition d'adaptation de ce processus en une démarche plus spécifique pour les entreprises libérées. Dans les sections suivantes nous montrerons comment nous avons mis en œuvre notre démarche au sein du Groupe Poult pour que les collaborateurs appréhendent mieux le concept de PLM ainsi que les tenants et les aboutissants de la mise en place d'une telle solution. Enfin, nous conclurons en étudiant les points forts et les points d'amélioration de notre proposition.

\section{Déploiement du PLM dans les entreprises}

\subsection{Démarche usuelle de déploiement d'une solution PLM}

Dans les entreprises, un PLM est souvent considéré comme une solution pour gérer tous les processus et les données associées, générées et distribuées durant la réalisation de tâches ou d'activités par différents acteurs (acteurs : personne physique ou outils logiciels) au cours du cycle de vie du produit de sa naissance jusqu'à sa fin de vie [STA 15]. Certaines entreprises, notamment les PMEPMI, ont quelques difficultés dans l'utilisation des systèmes PLM et n'exploitent pas totalement tout leur potentiel et de nombreuses informations et connaissances sont perdues ou exigent des efforts très importants pour être, malgré tout, préservées [EKA 09] [BRU 15]. Les plus importantes difficultés résident dans le manque de modèles facilement accessibles pour les entreprises pour représenter leur organisation, leurs processus et le cycle de vie de leurs produits mais aussi dans la démarche des éditeurs de PLM pour adapter et déployer leurs outils. Cette démarche est parfois déstabilisante pour les entreprises les moins bien préparées car ayant peu ou pas appréhendé les enjeux et les impacts du déploiement d'une solution PLM en leur sein [BAC 12a]. La démarche des éditeurs est généralement itérative, incrémentale et basée sur des modèles et des prototypes (figure 1). 

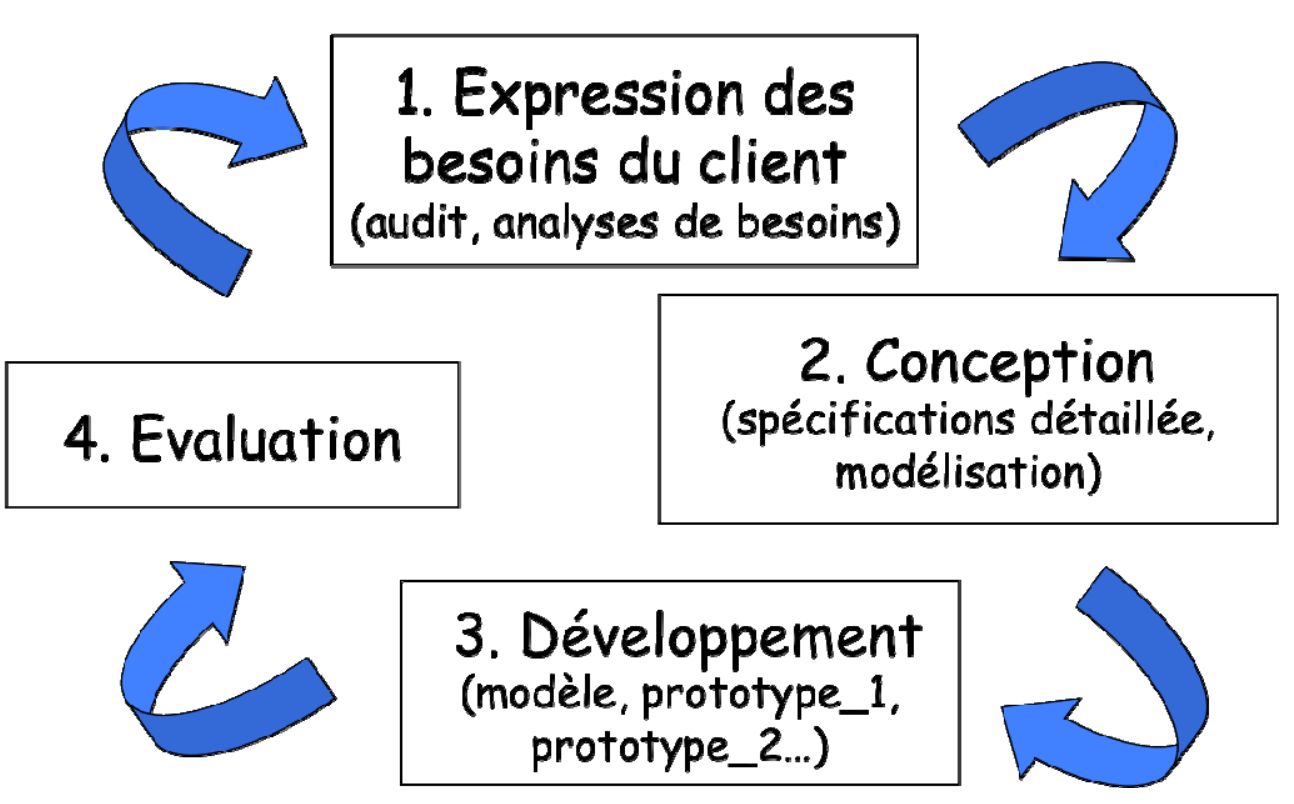

Figure 1. Démarche itérative et incrémentale d'analyse en vue du déploiement d'un PLM [BAC 12a]

Cette démarche descendante, assez standardisée, est utilisée pour modéliser et analyser les structures fonctionnelles, décisionnelles et organisationnelles du client mais n'assure par contre qu'une cohérence partielle entre les attentes du client et les fonctionnalités réelles de l'outil déployé. Les solutions logicielles étant bâties sur des « briques » standards il n'est pas toujours évident (et rentable) de les adapter à chaque client : le nombre d'itérations pour définir les attentes du client est très important et au bout d'un certain temps les retours d'expériences des acteurs ne sont plus toujours suffisamment pris en compte. Le problème réside donc dans la diversité et le caractère, malgré tout unique, de chaque solution à implanter. Vient s'ajouter à cela la difficulté liée aux visions et aux sémantiques parfois divergentes des acteurs : les clients/usagers sont motivés par les fonctionnalités et leur valeur ajoutée (et leur coût), alors que les éditeurs ont des objectifs structurels, technologiques et opérationnels. Ceci conduit souvent à des incompréhensions qui génèrent des difficultés au cours de la phase de validation des spécifications des fonctionnalités du logiciel.

\subsection{Proposition d'une démarche de déploiement adaptée - cas d'étude Poult}

La démarche décrite précédemment n'est pas totalement efficiente et cause souvent des retards significatifs sur les plannings initiaux. Pour réduire et optimiser l'analyse des besoins et des attentes des clients pour chaque nouveau projet et pour augmenter l'efficience de la procédure de déploiement d'un PLM, nous avons développé une procédure plus globale et centrée sur le cycle de vie de la solution logicielle dans le cadre de la thèse de Mylène Baczkowski [BAC 12a] (figure 2).

Notre objectif était de faciliter les analyses préliminaires des organisations et la caractérisation des attentes et des besoins des usagers chez le client. Notre démarche a été développée en partenariat avec LASCOM, un éditeur de solutions PLM français. Ce choix s'explique par le fait que LASCOM a déjà accompagné le Groupe POULT dans un précédent projet et que LASCOM a une forte expérience du PLM dans le secteur de l'agroalimentaire [LAS 18]. Nous n'avons pas pu (ou su) éviter qu'elle soit « orientée éditeur ». En d'autres termes, elle optimisait l'audit et l'analyse des besoins pour l'éditeur de PLM (surtout en termes de réduction des temps d'audit) et créait une forme de relation de dépendance entre le client et l'éditeur. Dans ce cas, les entreprises pouvaient être réticentes à l'endroit d'une telle démarche car préférant conserver une certaine autonomie, même relative, vis-à-vis des éditeurs. 


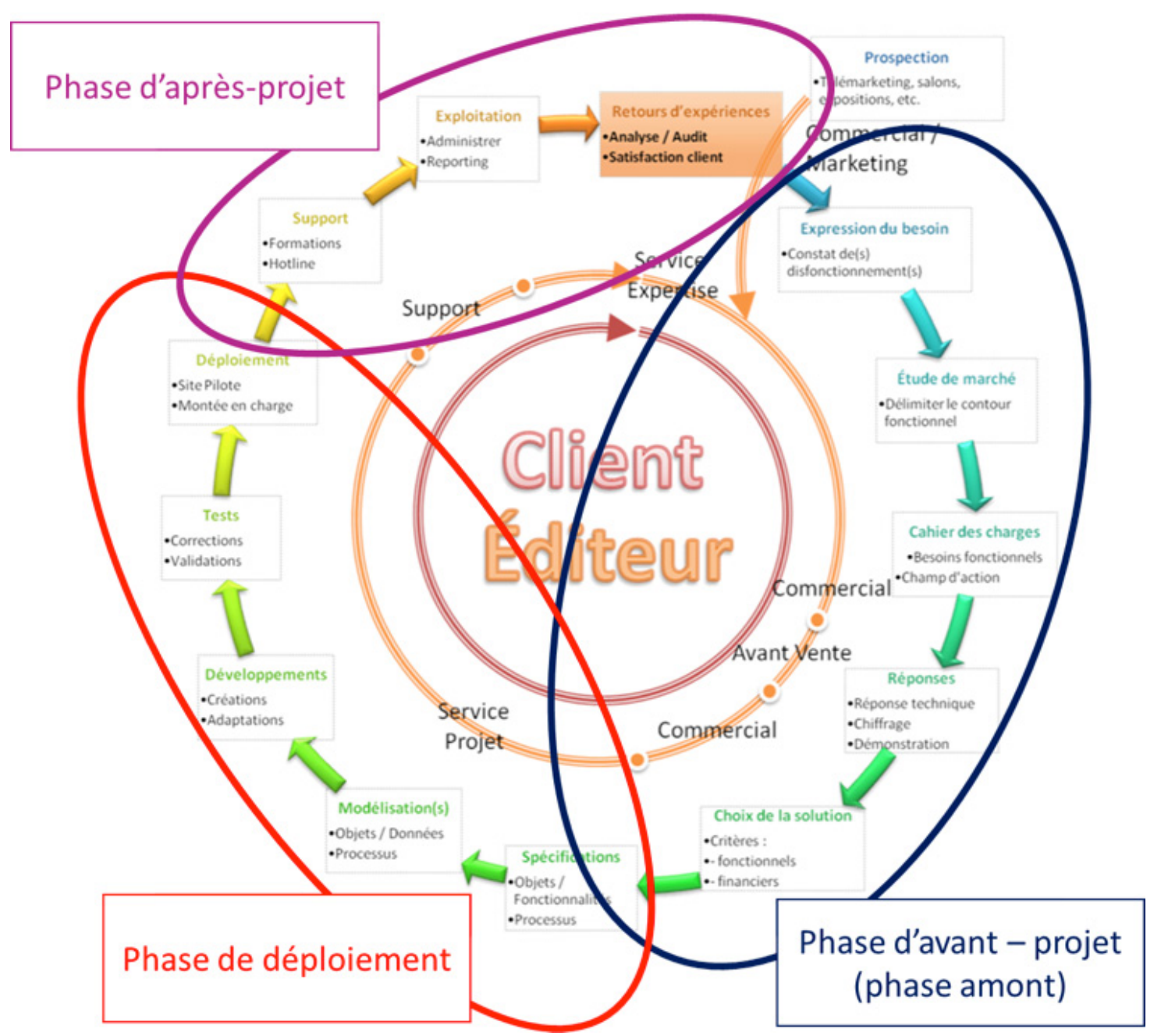

Figure 2. Procédure complète de déploiement d'un PLM avec implication du client [BAC 12a]

Généralement, les petites organisations comme les PME-PMI ou les organisations «particulières » ( « libérées» comme Poult par exemple) souhaitant avoir du temps pour comprendre et intégrer un système PLM, elles peuvent parfois se sentir «pressées » par les éditeurs et sont ainsi suspicieuses quant à leurs intentions. Pour limiter les effets néfastes d'une démarche trop centrée « éditeur ». C'est pour faire progresser notre vision du monde des entreprises libérées que nous avons souhaité travailler à la définition d'une nouvelle démarche en collaboration avec le Groupe Poult, une entreprise qui développe et produit des biscuits, qui avait depuis 2014 pour projet de déployer une solution PLM pour rendre son processus R\&D plus efficient.

\subsubsection{Contexte industriel : l’entreprise Poult, ses spécificités, ses ambitions}

Le Groupe Poult est leader des biscuits sucrés pour les marques distributeurs en France et a pour particularité d'avoir mis en place une organisation de type « entreprise libérée » il y a quelques années. Depuis 2014, Poult songeait à s'attaquer à l'export et a donc développé une stratégie en partie fondée sur une forte politique d'innovation et d'internationalisation. Pour soutenir cette stratégie le groupe a décidé de renforcer son pôle R\&D et d'optimiser son processus de conception de nouveaux produits notamment en repensant le SI par l'intégration d'un logiciel PLM. Pour le Groupe Poult l'objectif de cette intégration est de réduire son temps de réponse à une demande client et assurer une meilleure continuité entre les innovations « produit » et leur mise en production par :

- Un partage de l'information par la mise en place d'un référentiel produit unique permettant à l'entreprise de maintenir une information centralisée, fiable et à jour. En effet, à l'heure actuelle, chaque site/service et quasiment même chaque individu utilise ses propres outils « métiers », informations et données. 
- Une optimisation des processus en partageant et capitalisant sur les connaissances de l'entreprise pour poser les bases d'une forme "d'innovation continue » dans l'entreprise. Le système d'information actuel freine les innovations et leur internationalisation. Par exemple, les manquements dans le transfert d'informations issues de la veille réglementaire font que les données sont parfois insuffisamment précises pour répondre aux exigences réglementaires (européennes et internationales) en matière d'étiquetage, d'origines géographiques... A cela s'ajoute de nombreuses données éparpillées et dupliquées à différents endroits, ce qui augmente les temps de développement de certains projets.

- Une méthodologie structurée : le PLM contribuera à orchestrer les différentes phases de développement d'un produit en favorisant et accompagnant les innovations " produit » durant tout leur cycle de vie par la génération en automatique de la documentation associée au produit/projet. Aujourd'hui, des erreurs sont identifiées dans certains processus de conception par l'utilisation de documents ou d'informations erronés, des ressaisies d'informations...

- Une communication maîtrisée : le PLM permettra de contrôler le lancement du produit, de mieux maîtriser les plannings, de gérer les risques, d'améliorer la collaboration aussi bien en interne, entre les différents services du Groupe, qu'avec les acteurs extérieurs au Groupe.

L'un des enjeux de la mise en place d'une telle solution, relativement structurante, est la conservation de la culture d'entreprise et de cette forme de management chères à Poult : " l'entreprise libérée ». Notre recherche va donc s'attacher à définir les modalités et le phasage du déploiement d'une solution PLM dans un système managérial tel que celui du Groupe Poult et à accompagner le groupe avant, pendant et après l'implantation d'un outil PLM.

\subsubsection{Une démarche pour étudier « en interne » la pertinence du déploiement d'un PLM avant l'appel d'offres}

Le choix et le déploiement d'une application logicielle PLM peuvent provoquer une mise en tension entre le système d'information et les acteurs de l'entreprise car le logiciel impacte les pratiques des métiers et peut aller jusqu'à modifier des processus dans lesquels ils sont engagés. Tout le problème est dans cette tension qui peut conduire à deux formes d'actions : une adaptation du logiciel opérée par l'éditeur pour être plus en harmonie avec l'existant de l'entreprise (solution personnalisée) ou une adaptation des acteurs pour accepter une nouvelle pratique conforme au logiciel tel qu'il est fourni par l'éditeur (solution standard). Entre ces deux postures extrêmes, il peut y avoir des équilibres à trouver en conjuguant les deux formes d'actions avec une intensité justement dosée en fonction des niveaux de qualité de l'entreprise et du produit logiciel. Le Groupe Poult a déjà expérimenté cette posture médiane par le passé avec des éditeurs de logiciels. Les membres du groupe projet «PLM » qui avaient eu à travailler avec les éditeurs avaient eu la sensation (à tort ou à raison) que la dépendance à l'éditeur était trop rapide, trop forte et que les particularités de l'entreprise n'étaient pas assez prises en compte (solution trop « standard » et pas assez « personnalisée ») et le projet «PLM » avait été stoppé.

Le Groupe Poult préfère aujourd'hui travailler sur une approche plus centrée sur les acteurs vis-à-vis de cette mise en tension dans le but d'anticiper toute difficulté. Il souhaite donc avoir une réflexion en interne, en autonomie et très en amont de la phase de sélection d'une solution logicielle pour ainsi avoir du temps pour comprendre et intégrer ce qu'est un système PLM et les impacts que son implantation peut avoir sur l'entreprise. Poult a aussi choisi de se rapprocher de chercheurs du laboratoire IMS de l'Université de Bordeaux - Philippe Girard et Vincent Robin qui avaient déjà eu l'occasion de s'intéresser au processus de déploiement d'un PLM dans le cadre de la thèse de Mylène Baczkowski [BAC 12a] et sur la culture organisationnelle des entreprises [TOP 10]. La demande vis-àvis des chercheurs concerne la modélisation et la compréhension de la structure de l'entreprise libérée pour évaluer la pertinence de l'implantation d'une solution PLM et identifier les difficultés potentielles liées à un tel projet avant de revenir à nouveau vers les éditeurs. 
Dans cet article nous allons montrer comment nous avons proposé et mis en œuvre une démarche progressive (figure 3) pour accompagner les réflexions des membres de l'équipe projet PLM de Poult (responsables des départements $\mathrm{R} \& \mathrm{D}$, production, qualité, marketing, achats et Mlle Plo - doctorante CIFRE - Poult / Laboratoire IMS de 2015 à 2018).

Notre proposition se veut être une démarche permettant aux entreprises de comprendre en interne le concept de PLM, d'exprimer leurs besoins et leurs attentes vis-à-vis de ce type de solution logicielle et de définir leurs propres spécifications initiales en vue de la rédaction d'un cahier des charges et d'un appel d'offres en autonomie, avant de faire appel à un éditeur logiciel. Elle s'appuie sur l'expérience menée chez Poult durant les trois années de thèse et est composée de quatre étapes dont la finalité est de formaliser les besoins de l'entreprise avant la première rencontre avec un éditeur PLM (figure 3) :

- Etape 1 : Comprendre l'organisation du processus R\&D, pour modéliser l'organisation existante sans considérer une vision trop centrée sur le PLM et ses fonctionnalités,

- Etape 2 : Comprendre l'approche PLM, les enjeux et les impacts, pour apprécier et estimer les effets possibles de la mise en place d'un PLM dans l'entreprise,

- Etape 3 : Considérer les usagers dans le déploiement de la solution PLM pour favoriser l'acceptation de l'outil auprès des futurs usagers,

- Etape 4 : Formaliser les besoins et définir les spécifications préliminaires d'une solution qui pourrait être adaptée à l'entreprise, à ces besoins et à ceux des usagers.

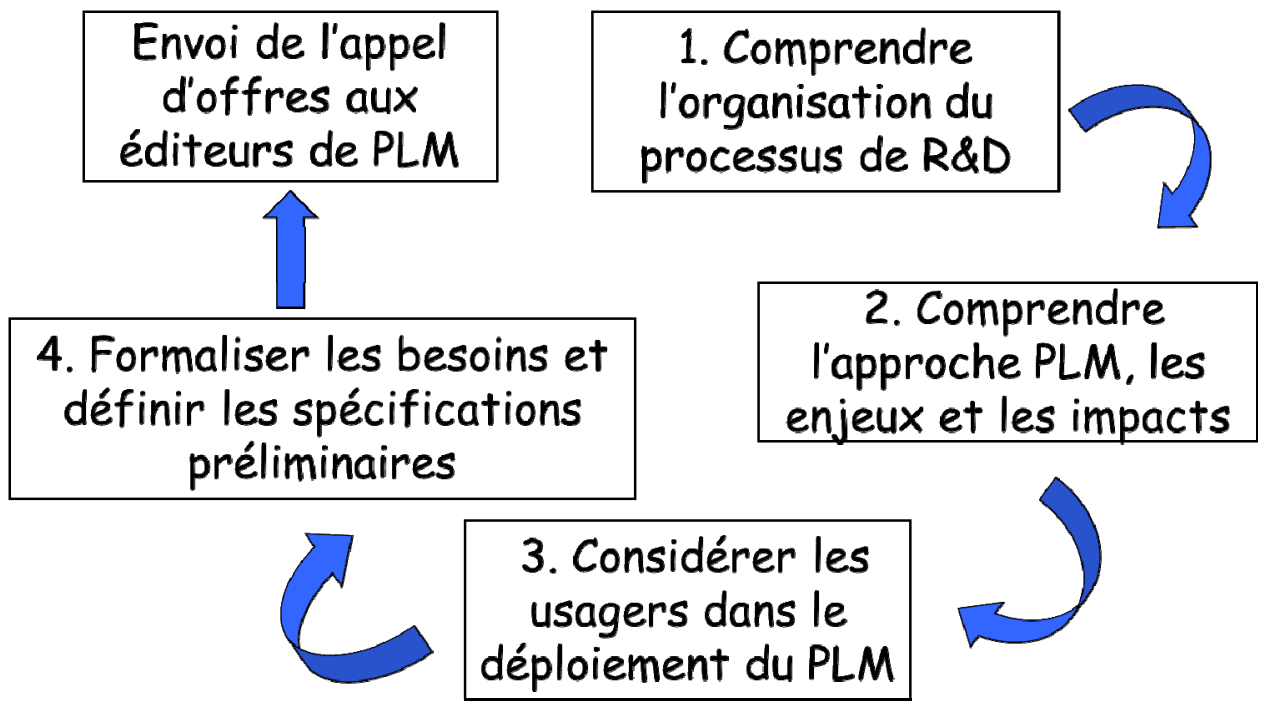

Figure 3. Démarche interne pour étudier la pertinence du déploiement d'une solution PLM dans une entreprise

Comme nous avons décrit, défini et explicité le processus de recherche qui nous a conduit à la définition de notre démarche [PIN 17], nous allons dans la suite de l'article nous focaliser sur la mise en œuvre pratique de notre proposition au sein du Groupe Poult.

\section{Etape 1 : Comprendre le processus R\&D}

Pour Chevallereau [CHE 09], les acteurs restent très souvent critiques par rapport à leurs systèmes d'information et ceci est principalement dû à la difficulté à produire un cahier des charges suffisamment détaillé pour les opérationnels et interprétable par les spécialistes des systèmes d'information. Les entreprises ont une volonté d'identifier et de définir au mieux l'ensemble de leurs procédures, des habitudes et des besoins de chacun des usagers au moment de la conception du 
système d'information, mais nous constatons que les méthodologies proposées restent très techniques et donc peu accessibles aux opérationnels. Ainsi, la première étape de notre démarche représente un véritable enjeu pour Poult car elle a pour principal objectif de fournir une spécification des besoins qui doit être aussi complète et documentée que possible, afin d'établir une communication suffisante entre les différents participants impliqués dans le projet, à la fois en interne (chez le client) et en externe (équipe projet avec les experts techniques côté éditeur).

Pour atteindre cet objectif, nous avons, tout d'abord, travaillé à l'analyse de la structuration actuelle des processus, des activités et des données chez Poult. Cette analyse s'est construite à travers la mise en place d'un questionnaire et d'interviews structurées, afin de faire émerger les besoins, les activités et rôle de chaque acteur, mais également les caractéristiques "personnelles » de chaque individu (connaissances, compétences...) (figures 4,5 et 6). Le choix de cette technique d'enquête (« questionnaire puis entretien ») a pour objectif de recueillir un maximum d'informations auprès des personnes interviewées. Avant la distribution du questionnaire, celui-ci a été testé auprès d'un ou plusieurs membres de l'équipe projet afin d'évaluer sa pertinence, les difficultés éventuelles que les interviewés auraient pour répondre aux questions, et d'identifier des erreurs tant sur le fond que sur la forme. Les entretiens préliminaires, " de test », ont permis de spécifier et compléter certaines questions. L'une des difficultés de l'entretien est d'amener la personne interviewée à « se raconter », il faut, par conséquent, poser quelques questions ouvertes et adopter une attitude empathique, avec une écoute active et des reformulations si nécessaire [GAV 11]. Ce questionnaire a été construit afin de recueillir un maximum d'informations sur les activités et tâches de chacun, les flux d'informations et les compétences et habitudes de chaque utilisateur. Nous nous sommes basés dans un premier temps sur les travaux de recherche de Roboam [ROB 93] et Dahmani et al. [DAH 13] pour passer d'une vision globale des décisions (macro-processus) (figure 4) à une vision locale de chaque centre de décision, où l'on représente l'enchainement détaillé des activités décisionnelles (figure 5). Mais cette démarche, principalement centrée sur les processus, n'était pas suffisante à la compréhension des besoins. Nous l'avons complétée par la mise en place de « persona » (figure 6a et 6b) [BAC 12b]. Le « persona » est un avatar des acteurs (futurs utilisateurs), qui est très proche de la réalité de l'usage qui sera fait de l'outil, et donc plus " précis » qu'un modèle d'usage et de comportement standard. Notre objectif était de déterminer les besoins réels des utilisateurs pour valider les exigences retenues, et s'assurer de la prise en compte de l'ensemble des besoins afin de maximiser la pertinence du logiciel, tout en intégrant les utilisateurs au projet d'informatisation.

Cette approche sur la base de questionnaires a mis en évidence le fait que même si l'entreprise est « libérée » cela ne signifie pas que les rôles sont assez précisément définis (voir tableau 1). En effet, même si les collaborateurs ont une certaine autonomie dans le système, chacun sait ce qu'il a à faire et le niveau décisionnel qui est le sien, celui des autres. Cela se traduit par une forme d'organisation fonctionnelle implicite et les rôles « chef de projet $R \& D$ », « magasiner », etc., sont clairement perçus et décrits par les acteurs mais leurs périmètres sont flous puisqu'il n'existe pas de fiche de poste. Ainsi, l'entreprise «libérée » peut être perçue comme une organisation assez horizontale et déhiérarchisée mais l'organisation fonctionnelle quant à elle ne disparait pas et est toujours bien présente plus ou moins implicitement. Même dans le contexte de l'entreprise « libérée », la modélisation des processus, et en particulier du processus R\&D, n’a globalement pas posé des difficultés,

Il est aussi à noter que nous avons fait une tentative de représentation du modèle décisionnel, avec le formalisme GRAI R\&D [GIR 06], qui n'a pas été concluante car nous n'avons pas pu mettre en évidence l'ensemble des relations et des liens (notamment informels) entre les acteurs. Ce modèle assez structurant demande à évoluer pour tenir compte du caractère déhiérarchisé de l'organisation, ce qui se rapproche d'autres travaux de Girard et Robin [GIR 04] sur les environnements de conception (nota : nous travaillons actuellement aux évolutions de ce formalisme et de ces travaux). 
2) Pourriez-vous décrire vos activités par ordre d'enchainement dans le cadre de la création ou de l'optimisation d'un produit/process? Pour chaque activité listée lors de la création ou de l'optimisation d'un produit/process, identifier l'horizon?Quel est votre horizon de prise de décision?

L’horizon correspond à la durée de la portée de la décision, la période quant à elle est l'intervalle de temps au bout duquel il est nécessaire de remettre en cause les décisions élaborées sur l'horizon considéré.

Pour illustrer ce concept très spécifique, prenons un exemple. L'activité faire un PDP (Programme Directeur de Production) à un niveau $\mathrm{H}=8$ mois et $\mathrm{P}=1$ mois. Cela signifie que le PDP est réalisé tous les mois à partir du carnet de commandes et en fonction du plan long terme de production. Le PDP définit le volume de production par produit pour 8 mois.

\begin{tabular}{|c|l|c|}
\hline \multicolumn{1}{|c|}{ Activités } & Horizon / Période (H/P) \\
\hline 1 & $\begin{array}{l}\text { Exemple Activité R\&D : Création de la fiche essai à donner au micro et prod la veille de l'essai avec code essai : détail recette + } \\
\text { code MP + données prod + traça MP par micro-ingrédients... }\end{array}$ & $\begin{array}{c}\text { H: durée du projet } \\
\text { essai est réalisée à chaque essai) }\end{array}$ \\
\hline
\end{tabular}

Figure 4. Première partie du questionnaire vision globale (macro-processus)

\section{) Identifier les interfaces entre les différentes activités listées lors de la création d'un nouveau produit.}

Je vous sollicite pour pouvoir modéliser vos réseaux de communication c'est-à dire :

- Quelles sont les informations que vouz recevez ? De qui ? Et appréciation de l'information?

- Quelles sont les informations que vous transmettez? A qui ?

\begin{tabular}{|c|c|c|c|c|c|c|c|c|c|}
\hline \multicolumn{5}{|c|}{ Informations utilisées } & \multirow[b]{2}{*}{ Activités } & \multicolumn{4}{|c|}{ Informations transmises } \\
\hline $\begin{array}{l}\text { Origine (indiquer services } \\
\text { + nom de la personne / } \\
\text { prestataires extérieur en } \\
\text { indiquant laboratoire ou } \\
\text { client ou fournisseur ou } \\
\text { autre à préciser) }\end{array}$ & $\begin{array}{l}\text { Contenu de } \\
\text { l'information }\end{array}$ & $\begin{array}{l}\text { A cocher si } \\
\text { information } \\
\text { écrite }\end{array}$ & $\begin{array}{l}\text { A cocher si } \\
\text { information } \\
\text { orale }\end{array}$ & $\begin{array}{l}\text { Appréciation de } \\
\text { la comunication : } \\
\text { excellente / } \\
\text { correcte / à } \\
\text { améliorer / } \\
\text { inexistante / très } \\
\text { mauvaise }\end{array}$ & & \begin{tabular}{|} 
Destination (indiquer \\
services + nom de la \\
personne / \\
prestataires extérieur en \\
indiquant laboratoire ou \\
client ou fournisseur)
\end{tabular} & $\begin{array}{l}\text { Contenu de } \\
\text { l'information }\end{array}$ & $\begin{array}{l}\text { A cocher si } \\
\text { information } \\
\text { écrite }\end{array}$ & $\begin{array}{l}\text { A cocher si } \\
\text { information } \\
\text { orale }\end{array}$ \\
\hline
\end{tabular}

Figure 5. Deuxième partie du questionnaire vision locale (détail des activités sous forme de processus)

Je vous sollicite pour connaître les types de documents que vous manipulez dans votre travail quotidien ou occasionnel à partir des activités listées.

Il est indispensable de pouvoir cartographier la gestion documentaire. Car à l'heure actuelle, il y a clairement un manque de visibilité lié à l'éparpillement des documents sur différents supports, à la fois les serveurs partagés (U, S pour le Sud Ouest), les disques personnels (W), les intranets (GED, Trace One...), la boîte de messagerie et à l'utilisation d'une multitude d'outils aux usages qui ne sont pas toujours bien rationalisés. La première question à se poser est : quel outil pour quelle utilisation. La cartographie ci-dessous fait le point sur les outils et leur usage.

Cette cartographie, va permettre d'identifier le fonctionnement de chaque zone de stockage des documents (utilisation, critères de stockage...)

Pour cela, il vous suffit de renseigner le tableau ci dessous en précisant en fonctions des activités :

- le type de document (le nom utilisé couramment)

-un complément sur ce type de document (un autre nom utilisé par exemple)

-la provenance de ce document (interne = s'il est réalisé dans l'entreprise ou externe)

-la localisation de ce document (où le document est stocké)

- la fréquence à laquelle vous manipulez ce document

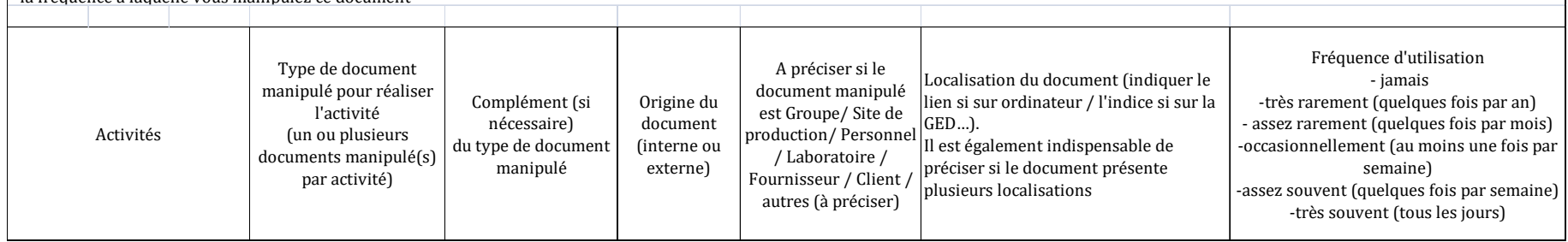

Figure 6a. Troisième partie du questionnaire « mise en place des persona » - partie 1 (généralités sur les documents)

Je vous sollicite pour mieux connaître votre cadre d'utilisation de ces documents.

Pour cela, il vous suffit de renseigner le tableau suivant en illustrant un cas d'utilisation par une phrase expliquant le contexte (quand et pourquoi). Ensuite il ne vous restera plus qu'à enregistrer le fichier et me le renvoyer. Merci de votre participation.

\begin{tabular}{|c|c|c|c|c|c|c|c|c|}
\hline Activités & $\begin{array}{c}\text { Type de } \\
\text { document }\end{array}$ & $\begin{array}{l}\text { Fréquence } \\
\text { d'utilisation }\end{array}$ & $\begin{array}{l}\text { Vous créez ce type } \\
\text { de document } \\
\text { (création) }\end{array}$ & $\begin{array}{l}\text { Vous modifiez ce type } \\
\text { de document (éditer) }\end{array}$ & $\begin{array}{l}\text { Vous êtes chargés de } \\
\text { mettre en application } \\
\text { ce type de document } \\
\text { (mise en application) }\end{array}$ & $\begin{array}{c}\text { Vous validez ce type } \\
\text { de document } \\
\text { (approuver) }\end{array}$ & $\begin{array}{c}\text { Vous envoyez en } \\
\text { diffusion } \\
\text { ce type de } \\
\text { document (diffuser) }\end{array}$ & $\begin{array}{c}\text { Vous } \\
\text { consultez ce } \\
\text { document } \\
\text { pour } \\
\text { information } \\
\text { (consulter) }\end{array}$ \\
\hline
\end{tabular}

Figure 6b. Troisième partie du questionnaire " mise en place des persona » - partie 2 (actions sur les documents) 


\begin{tabular}{|c|c|c|}
\hline Activité & Tâche (processus métier) & Acteur impliqué \\
\hline \multirow{3}{*}{$\begin{array}{l}\text { Appel d'offres d'un } \\
\text { client ou interne (projet } \\
\text { "Poult ") }\end{array}$} & $\begin{array}{l}\text { Demande de développement d'un nouveau produit } \\
\text { Demande de validation }\end{array}$ & Client \\
\hline & Etude de faisabilité & $\begin{array}{l}\text { "Famille Produit" (R\&D, marketing, } \\
\text { achats, production) }\end{array}$ \\
\hline & Validation étude de faisabilité & \\
\hline \multirow{3}{*}{ Revue interne } & Définition succincte du produit & Marketing \\
\hline & Définition succincte du packaging & Développement packaging \\
\hline & Validation des définitions préliminaires & "Famille Produit" \\
\hline \multirow{4}{*}{ Premiers tests } & Définition des tests préliminaires & $\begin{array}{l}\text { Chef de projet } R \& D \text {, responsable de la } \\
\text { production }\end{array}$ \\
\hline & Achats des ingrédients spécifiques & Chef de projet R\&D, achats \\
\hline & Réception des ingrédients spécifiques & Assistant $\mathrm{R} \& \mathrm{D}$, magasinier \\
\hline & Définition des tests (formulation de la recette) & \\
\hline \multirow{4}{*}{$\begin{array}{l}\text { Tests en laboratoire et } \\
\text { tests industriels }\end{array}$} & Calcul du prix de revient & Chef de projet R\&D \\
\hline & $\begin{array}{l}\text { Test de la recette } \\
\text { Contrôle des produits }\end{array}$ & $\begin{array}{l}\text { Chef de projet } R \& D \text {, responsable de la } \\
\text { production }\end{array}$ \\
\hline & Dégustations (en interne) & Chef de projet R\&D, Assistant R\&D \\
\hline & Validation des dégustations & "Famille Produit" \\
\hline \multirow{4}{*}{ Validation interne } & Validation de la conformité & Chef de projet R\&D, Qualité \\
\hline & Edition des cartes d'étiquetage provisoires & Chef de projet R\&D \\
\hline & \multirow{2}{*}{$\begin{array}{l}\text { Analyse du prix de revient global } \\
\text { Analyses physicochimiques }\end{array}$} & Achats, contrôleur de gestion \\
\hline & & Laboratoire Externe \\
\hline $\begin{array}{l}\text { Echantillons pour le } \\
\text { client }\end{array}$ & $\begin{array}{l}\text { Production d'échantillons pour le client } \\
\text { Validation des échantillons par le client } \\
\text { Envoi des échantillons au client }\end{array}$ & Chef de projet R\&D, Assistant R\&D \\
\hline & Acceptation du client & Client \\
\hline $\begin{array}{l}\text { Validation finale par le } \\
\text { client } \\
\text { Industrialisation }\end{array}$ & $\begin{array}{l}\text { Jurys internes et externes } \\
\text { Validation finale de la recette }\end{array}$ & Client \\
\hline
\end{tabular}

Tableau 1. Description préliminaire des activités, des tâches et des acteurs impliqués dans le processus R\&D

A travers les réponses, nous avons fait un diagnostic de la situation initiale et réfléchi à la priorisation et au choix des axes d'évolution du PLM. Le questionnaire a permis de mettre en évidence les activités et les tâches principales du processus de R\&D de Poult, les acteurs impliqués (synthèse en tableau 1), et d'aboutir à un modèle de processus. Nous avons aussi pu avoir une vision assez fine des méthodes et habitudes de travail des collaborateurs par le biais des réponses liées aux questions relatives aux personas. Nous avons établi des fiches individuelles (non présentées ici), qui pourront éventuellement nous aider à anticiper les difficultés des collaborateurs, vis-à-vis de l'outil, et donc nous aider à mieux cibler le contenu des formations que nous mettrons en place avant et pendant le déploiement de l'outil. La phase de déploiement, et donc les étapes de formation, seront décrites dans de prochains articles, dès lors que la sélection de l'éditeur logiciel sera faite.

\section{Etape 2 : Comprendre l'approche PLM, les enjeux et les impacts}

Comme le montrent Bruno et al. dans leurs travaux [BRU 15], la gestion efficace du cycle de vie du produit devient une nécessité pour les entreprises. Cette gestion passe par une structuration cohérente de la donnée afin de représenter et relier différents éléments d'information dans le but d'améliorer les performances, en réduisant notamment le temps de recherche des informations. Les travaux de Bruno et al. ont abouti à la définition d'une ontologie de référence PLM, dans le but de gérer au mieux les cycles de vie de produit dans des domaines industriels diversifiés. Le modèle ainsi défini est généralisable et utilisable pour de nombreux industriels. Sur la base des éléments collectés, à travers les questionnaires et interviews, nous avons été en mesure de bâtir un premier modèle de données propre à Poult sous la forme d'un diagramme UML, inspiré de celui proposé par Bruno et al.. Notre objectif était de faire en sorte que ce modèle constitue un véritable support au projet et un véritable moteur de réflexion sur la gestion et la coordination de la donnée et des informations qui seront gérées 
dans le futur logiciel PLM. Ainsi, nous avons présenté les concepts PLM et notre diagramme UML à l'équipe projet dans le but d'accompagner les membres de l'équipe dans leur passage d'une vision « processus classique » à une vision " processus/PLM ». Le diagramme UML s'est avéré être un bon support d'échange entre les collaborateurs pour spécifier, définir et structurer la gestion de la donnée entre les acteurs, et plus largement le processus R\&D. La figure 7 est la synthèse des travaux de l'équipe projet. Toutes les classes du diagramme UML appliquées à un projet PLM développés par Bruno et al. ont été conservé, et dix nouvelles classes ont été introduites afin de représenter les particularités du Groupe Poult et de son métier : l'agroalimentaire (tableau 2). Pour compléter, cette approche «théorique » et aider les acteurs à percevoir la traduction "informatique » du modèle théorique nous leur avons présenté un panel de solutions PLM pour l'agroalimentaire que nous avions identifiées suite à un benchmark des logiciels existants [PIN 17].

L'organisation de type "libérée » de l'entreprise Poult n'a pas d'influence sur les classes du diagramme puisqu'il n'est finalement qu'une représentation globale d'une organisation relativement à ses produits et ses processus et que les produits et les processus de Poult sont somme toute classiques et proches de ceux qui ont cours dans l'industrie agroalimentaire. Par contre, la représentation des flux d'information et de façon concomitante la représentation des cycles de vie (des données) sont plus complexes à tracer et en particulier les évènements clés permettant le passage d'un statut à un autre. Les processus décisionnaires étant assez flous (les rôles fonctionnels sont assez facilement identifiés mais ils ne sont pas forcément liés à des "capacités décisionnelle) 》 et il n'est donc pas simple de formaliser précisément « qui a la capacité de faire quoi » dans les processus. Le diagramme de classes a la vertu de poser les éléments de base des activités des acteurs mais il ne permet pas de mettre en évidence « qui peut faire quoi ? » et « sur quoi ? ». C'est l'une des limites les plus fortes de la démarche mais les acteurs de chez Poult n'ont pas été en mesure (ou n'ont pas souhaité) trop rentrer dans le détail des processus décisionnaires discrétionnaires en préférant rester sur les grands points d'étape décisionnaires que sont les comités de pilotage (aux périmètres eux aussi variables en fonction des activités concernées).

\begin{tabular}{|c|c|}
\hline Classe & Description \\
\hline $\begin{array}{l}\text { Process } \\
\text { (complète la classe } \\
\text { Produit) }\end{array}$ & $\begin{array}{l}\text { Le service R\&D peut développer un nouveau produit (i.e. } \\
\text { nouveau biscuit) ou un nouveau process de fabrication. Une } \\
\text { classe « Process » a donc été créé pour clairement dissocier } \\
\text { ces deux types de projets. }\end{array}$ \\
\hline $\begin{array}{l}\text { Site de fabrication } \\
\text { (complète la classe } \\
\text { Client) }\end{array}$ & $\begin{array}{l}\text { La conception d'un produit ou d'un process peut être initiée } \\
\text { par un client pour par un site de production de Poult. Une } \\
\text { classe «Site de fabrication » a donc été créée pour pouvoir } \\
\text { faire apparaitre ces deux types de donneurs d'ordre. }\end{array}$ \\
\hline $\begin{array}{l}\text { Recette et Composant } \\
\text { (pour préciser la classe } \\
\text { Produit) }\end{array}$ & $\begin{array}{l}\text { Les produits de Poult sont définis par le biais de recettes et } \\
\text { chaque recette regroupe plusieurs composants. C'est pour } \\
\text { cette raison que la classe " composant produit » dans le } \\
\text { modèle de Bruno a évolué en } 2 \text { classes : "recette " et } \\
\text { " composant ». Un produit possède une seule et unique } \\
\text { recette, mais plusieurs composants, le même composant } \\
\text { pouvant être utilisé dans plusieurs recettes. }\end{array}$ \\
\hline $\begin{array}{l}\text { Matière Première et } \\
\text { Ingrédient (remplacent } \\
\text { " matériaux " dans le } \\
\text { modèle de Bruno) }\end{array}$ & $\begin{array}{l}\text { Dans l'agroalimentaire le terme " matière première » est } \\
\text { préférable à celui de «matériaux ». Une matière première } \\
\text { peut être composée de plusieurs ingrédients. La } \\
\text { combinaison des ingrédients est une donnée important dans } \\
\text { le cadre du respect des normes agroalimentaires. }\end{array}$ \\
\hline Emballage & $\begin{array}{l}\text { Un produit est un ensemble composé d'un ou plusieurs } \\
\text { biscuits et d'un emballage spécifique. Poult gère ses } \\
\text { biscuits et ses emballages séparément d'où la création } \\
\text { d'une classe emballage. }\end{array}$ \\
\hline $\begin{array}{l}\text { Caractéristiques de } \\
\text { l'emballage }\end{array}$ & Les différentes caractéristiques de l’emballage. \\
\hline Fournisseur & $\begin{array}{l}\text { Dans l'agroalimentaire toutes les matières premières } \\
\text { doivent être contrôlées. L'un des contrôles concerne la } \\
\text { traçabilité des produits, l'identification et le suivi des } \\
\text { fournisseurs sont donc primordiaux. }\end{array}$ \\
\hline $\begin{array}{l}\text { Caractéristiques } \\
\text { Machine }\end{array}$ & $\begin{array}{l}\text { Les règles de l'agroalimentaire obligent de gérer les } \\
\text { données relatives aux machines utilisées pour la production } \\
\text { des biscuits. Cette classe répond aux exigences d'assurance } \\
\text { qualité pour le client final du processus de fabrication dans } \\
\text { son ensemble. }\end{array}$ \\
\hline
\end{tabular}

Tableau 2. Description des classes et concepts additionnels adaptés pour Poult (agro-industrie) 


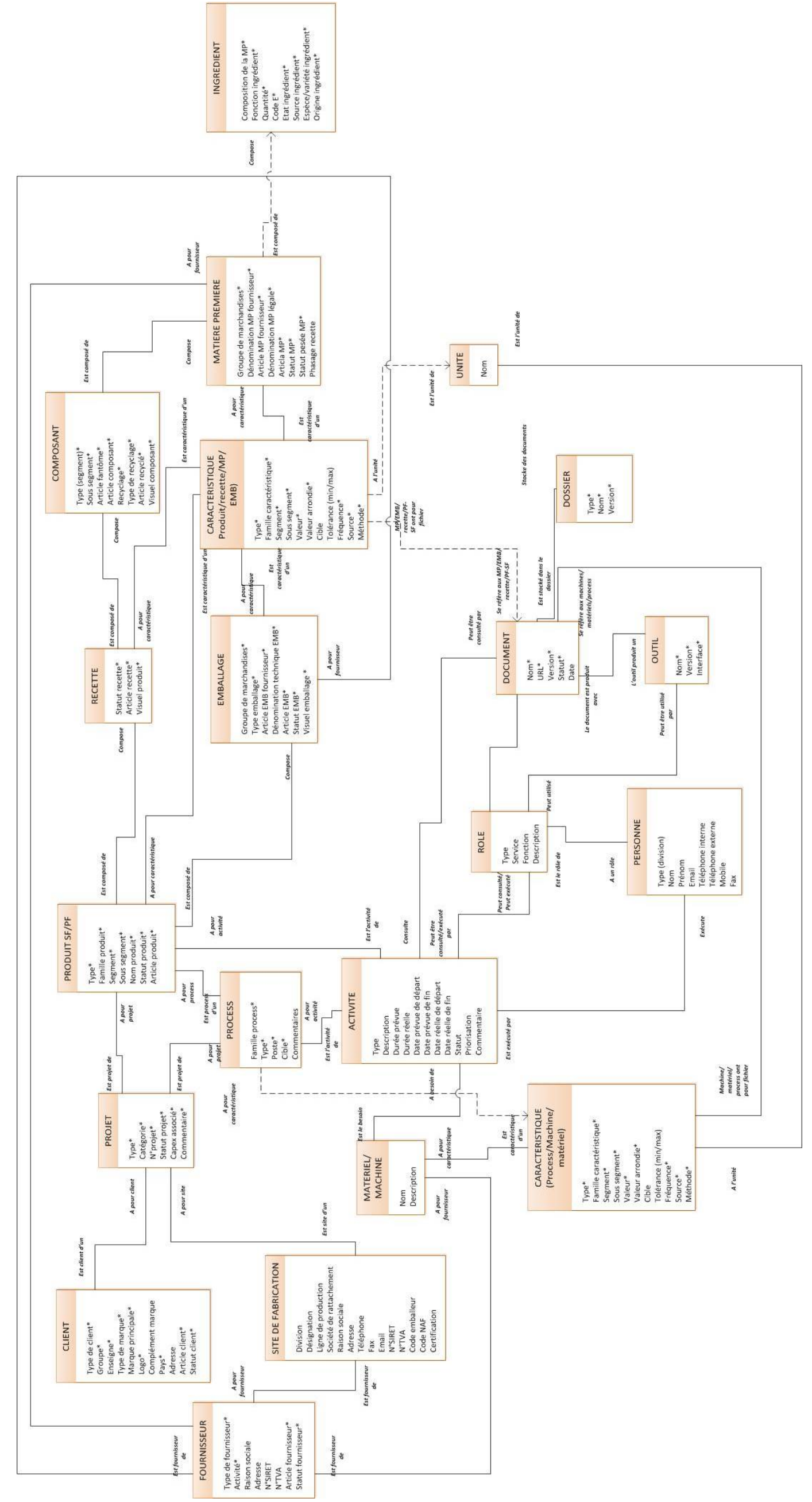

Figure 7. Diagramme UML de la conception PLM adapté au Groupe Poult 


\section{Etape 3 : Considérer les usagers dans le déploiement du PLM}

Le diagramme de classes UML adapté à Poult est le reflet de ce que les membres de l'équipe projet ont compris du PLM. Leur vision a peu à peu évolué d'une vision globale du processus de R\&D à une vision plus opérationnelle intégrant les concepts du PLM. Grâce à une telle représentation c'est l'architecture du système PLM qui est apparue plus clairement aux collaborateurs. Comme le déploiement et l'implémentation effective d'une solution ne se résume pas seulement à la sélection et à l'installation d'un logiciel mais passe, aussi et surtout, par l'acceptation des usagers de ladite solution, nous avons décidé de nous focaliser, au cours de cette étape, sur les usagers. La compréhension des besoins et des attentes des futurs utilisateurs est une phase critique dans le déploiement de telles solutions. Malheureusement, nombreux sont les travaux de recherche relatifs au management de projets « logiciels » qui ne considèrent pas correctement les attentes des utilisateurs et négligent la façon d'aborder et de gérer ces aspects auprès des chefs de projet [ROS 07]. Cette étape cruciale dépend de la capacité du chef de projet à comprendre et considérer les attentes des usagers [PET 08], et d'analyser, coordonner et piloter la collaboration entre les différentes parties prenantes du projet : les décisionnaires stratégiques et tactiques, les chefs de projets « produit », les concepteurs, les experts de différentes disciplines, les partenaires extérieurs, etc. Les visions et les sémantiques entre tous ces usagers sont parfois très différentes, ce qui conduit à des incompréhensions et génère des difficultés, en particulier au moment de la phase de spécification des fonctionnalités de la solution logicielle. Pour limiter ces problèmes nous avons développé, dès 2012, une démarche orientée " éditeur » pour faciliter les échanges entre les éditeurs de PLM et les entreprises souhaitant implanter une telle solution [BAC 12a]. Notre objectif était de rendre l'analyse préliminaire des besoins et des attentes plus aisée, en basant les échanges sur des modèles de type mindmap et par l'utilisation de personas. La mindmap initiale se structure en 4 branches et l'éditeur initialise une carte heuristique spécifique pour chaque client, dès le démarrage d'un projet d'implantation (figure 8) :

- « Modélisation de l'entreprise »: qui regroupe les informations concernant l'entreprise cliente (organisation structurelle et fonctionnelle, acteurs/utilisateurs impliqués dans le projet, etc.). Cette branche évolue et devient de plus en plus précise au cours de l'évolution du projet et de la récupération d'informations.

- «Suivi des contributions »: qui permet au chef de projet (coté éditeur) d'avoir une trace de chaque action réalisée au cours du projet. Cette branche a pour but de permettre de suivre chaque évolution du projet en spécifiant « qui a décidé de quoi ? », pour ainsi mieux tracer les décisions qui ont amené certains choix et spécifications de l'outil.

- «Suivi des livrables » : regroupe tous les documents qui ont été produit tout au long du projet. Cette branche aide l'éditeur et le client à juger de l'état d'avancement du projet relativement aux jalons communs, fixés au départ du projet.

- «Définitions des applications » : est la branche dans laquelle se trouvent toutes les versions de la solution logicielle développée pour le client au cours du projet.

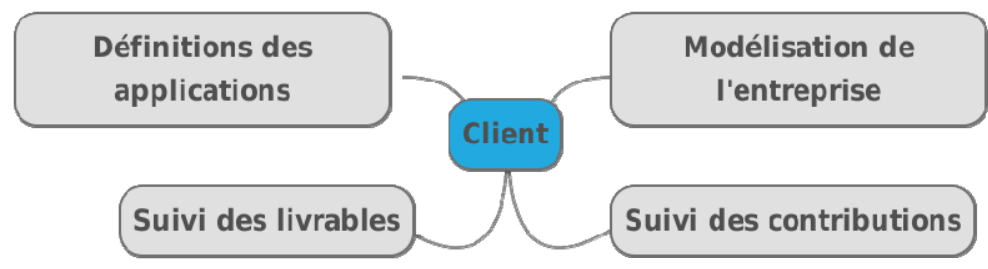

Figure 8. Architecture globale de la carte heuristique dédiée à un client 
Chaque acteur (éditeur ou client), qui est impliqué dans le projet, va participer, à son niveau, à la construction progressive des branches. Les informations concernant le client et le cycle de vie du projet (activités et documents associés) sont dans la mindmap et chaque étape du développement de la solution est capitalisée et réutilisable. Des relations existent entre les éléments de chaque branche pour obtenir une vision dynamique globale du système étudié. Une telle représentation rend les relations entre l'éditeur logiciel et son client plus simples [BAC 12b] et chaque carte est unique, en ce sens qu'elle est dédiée à un projet pour un client. Quand la solution PLM est déployée, il est assez aisé de la maintenir car l'éditeur à normalement toutes les données du client sur la mindmap et une action/évolution sur la solution est aussitôt répercutée sur la mindmap et réciproquement.

Dans le cadre du travail de recherche au sein du groupe Poult nous avons décidé d'inverser la proposition en faisant en sorte que cela soit le client et non l'éditeur qui construise la carte heuristique et l'initialise. L'objectif principal est de faire en sorte que le client puisse s'approprier la démarche, soit maître d'œuvre de son projet dès le départ et puisse éventuellement lui-même définir ses besoins et les spécifications qu'il souhaiterait. Cet objectif répondait aussi à la demande du Groupe Poult qui suite à sa première expérience avec les éditeurs logiciels voulait être beaucoup plus autonome dans son projet.

Ainsi, suite aux interviews, nous avons pu obtenir une « modélisation de l'entreprise » avec :

- Un modèle du Groupe Poult pour aider les acteurs à se situer dans le Groupe et à entrer dans une démarche de modélisation (carte heuristique - figure 9). Ce formalisme est assez pauvre en termes de données et de connaissances associées mais il a l'avantage d'être accessible pour tous les acteurs, même non experts, ce qui participe de leur intégration dans la démarche.

- Un modèle décisionnel : niveau stratégique (grille GRAI et réseaux GRAI, non présentés ici),

- Un modèle organisationnel : niveau tactique (BPMN, non présenté ici),

- Un modèle centré sur les acteurs : niveau opérationnel (Persona, non présenté ici).

Toutes les données récoltées lors des interviews ont été exploité et l'intégration de plusieurs formalismes de représentation a aidé les acteurs à rentrer dans le projet et à s'y impliquer. De plus, l'expérience a montré que cela permet aussi à l'éditeur sélectionné d'avoir une vue d'ensemble du « système » Poult, de ses acteurs et de leurs besoins. Les autres branches de la mindmap ne sont pas encore complétées car elles concernent l'éditeur PLM mais elles le seront avec celui-ci lorsqu'il sera sélectionné.

Suite à ces travaux un cahier des charges a été rédigé (environ 30 pages) et un appel d'offres a été lancé. Le cahier des charges reprenait l'ensemble des modèles (les données, les activités, les processus et l'organisation étaient spécifiés) et les besoins par classes d'acteurs étaient aussi définis « métier par métier » et « processus par processus ». C'est éléments étaient mis en parallèle des fonctionnalités des PLM identifiés dans notre benchmark. Plusieurs éditeurs ont répondu et ont tous été surpris par la qualité du travail de réflexion déjà mené et par la qualité, la richesse et la maturité que cette démarche «d'anticipation» apportait aux échanges client/éditeur. Suite aux nombreux échanges que nous avons pu avoir avec les éditeurs, il ressort que les besoins exprimés par les acteurs dans le cahier des charges sont souvent très pragmatiques et finalement «assez classiques ». En effet, comme nous l'avons déjà souligné, les produits et les processus industriels de Poult étant très proches de ceux des autres entreprises de l'agroalimentaire, les solutions PLM existantes pour ce secteur couvraient une grande partie des besoins. Le point de différentiation avec les autres entreprises concerne le besoin d'avoir des processus moins individualisés (non centrés sur une personne) mais plus collaboratifs. D'un point de vue «technique» la réponse apportée par les éditeurs est souvent la création d'un acteur de type «groupe » qui est activable dans les processus en fonction des besoins et qui permet que cela soit un groupe d'acteurs qui décide de façon collégiale. 
Malheureusement, suite à un rachat du Groupe Poult par un fond d'investissement, le projet PLM est aujourd'hui stoppé pour une durée indéterminée et nous ne sommes donc pas en mesure de présenter de réels retours d'expériences quant à la sélection et au déploiement d'une solution logicielle.

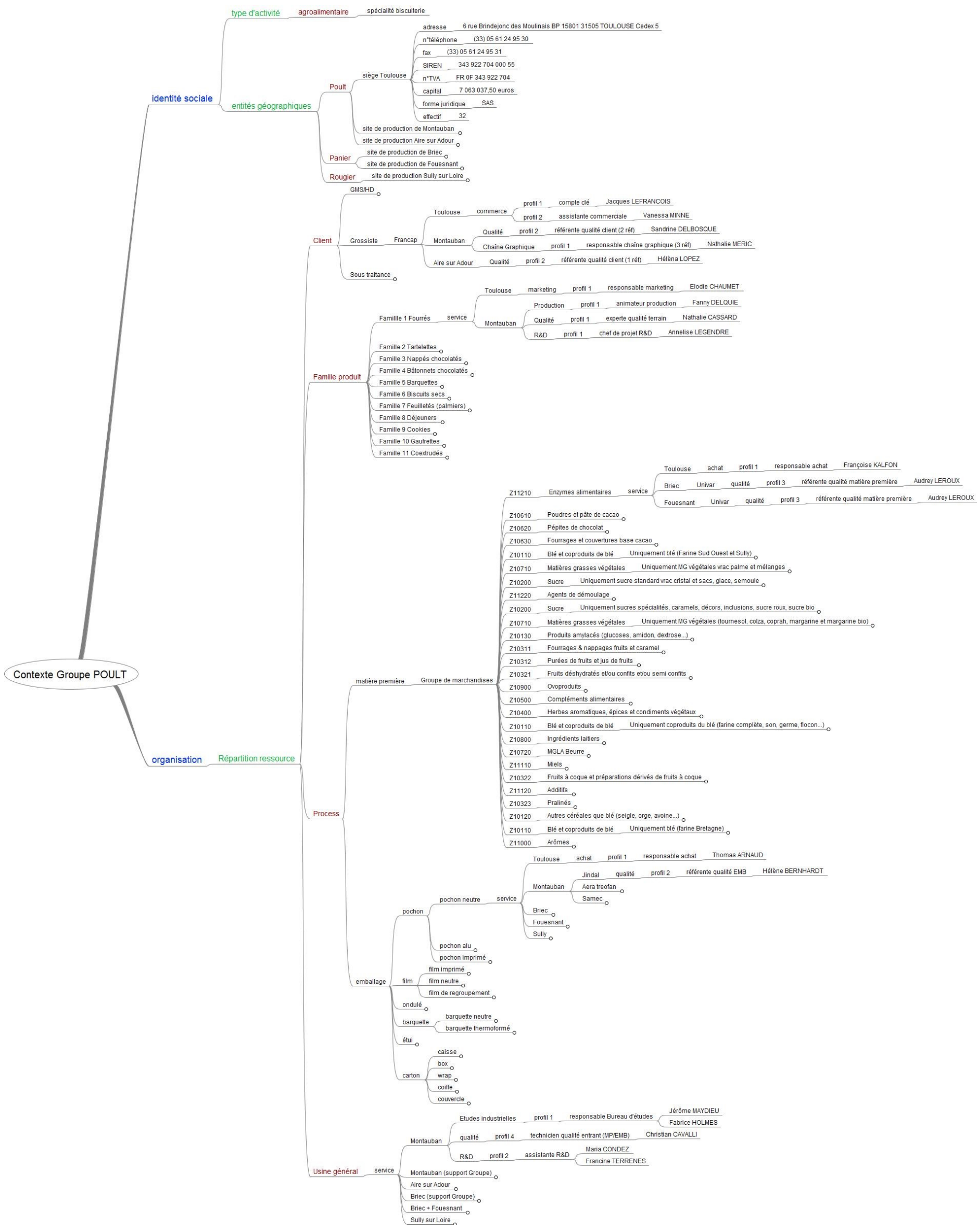

Figure 9. Modélisation du site de production de Montauban 


\section{Conclusion}

En partant des recherches de Stark [STA 15] et Cantamessa et al. [CAN 12] qui ont identifié les impacts du déploiement de l'outil PLM, à la fois sur les acteurs, les processus organisationnels et la stratégie de l'entreprise, notre démarche, en vue de l'implantation d'un outil PLM au sein de l'entreprise Poult, a consisté à mener une réflexion progressive en interne, sans faire appel à un éditeur logiciel, pour modéliser les processus et définir les besoins (figure 3). Les données collectées par le biais des questionnaires et interviews nous ont permis de modéliser le processus de « développement d'un nouveau produit suite à un besoin interne ou client » et celui de " mise en marché d'un nouveau produit suite à une validation du client ", et de mettre en évidence la structure organisationnelle de l'entreprise représentée, à la fois par le diagramme de classe de Poult (figure 7) et une mindmap (figure 9). D'autres formalismes auraient pu nous aider à faire ce travail mais, dans le contexte de l'entreprise Poult, il nous a été demandé d'impliquer tous les collaborateurs qui prennent part, de près ou de loin, dans les processus étudiés pour que chacun s'approprie la démarche. Ce souci de faire participer un grand nombre de personnes nous a donc conduit à arrêter notre choix sur un formalisme compréhensible par tous et ce sont donc le diagramme UML (ce qui peut paraitre surprenant) et la mindmap qui ont fait consensus. Ces formalismes ont pour effet d'aider les collaborateurs à mieux comprendre le système. Le diagramme UML a été perçu comme un outil de représentation riche et pertinent car il permet d'avoir, sur une seule figure, l'ensemble des éléments du système, leurs attributs spécifiques et leurs interactions. La mindmap, quant à elle, a eu pour vocation d'aider à spécifier plus en avant les profils, attentes et besoins de tous les acteurs qui contribuent, de près ou de loin, aux processus de développement du produit. Elle participe aussi à l'identification des différents modes de structuration organisationnelle présents sur le site et à l'évaluation de leur pertinence et des dysfonctionnements éventuels. Par exemple, et sans tous les citer ici, nous avons constaté qu'il y avait quelques dysfonctionnements dans la coordination des activités du processus de développement :

- Lors de la formulation d'une recette, les exigences clients et règlementaires ne sont pas toujours communiquées au chef de projet $\mathrm{R} \& \mathrm{D}$, alors qu'il devrait en être destinataire, en tant que responsable du développement.

- La fiabilisation du référentiel produit (matières premières fournisseurs) n'est pas assurée et pas centralisée. Le référentiel n'est pas donc pas toujours fiable et pas toujours à jour.

- Des données clés ne sont pas totalement tracées, et les flux informationnels et décisionnels ne sont pas totalement coordonnés et assurés.

Au-delà de la modélisation et de la clarification des processus et des flux, nous sommes aussi en train de mieux formaliser les processus collaboratifs et les processus métier qui devront être fiabilisés dans le PLM [EKA 08]. Pour le processus métier « formulation recette » (tableau 1) par exemple, nous avons mieux cerné ses spécificités et l'expertise nécessaire à sa réalisation. Le rôle et la place du chef de projet $R \& D$, en charge de la formulation de la recette, sont apparus plus clairement, ses besoins et ses compétences aussi (expertise à la fois des exigences client et réglementaires et une connaissance métier). A partir de là, nous sommes en mesure d'identifier les éléments porteurs de connaissance et les supports à la décision, ainsi que les « règles métiers ». Les données manipulées à capitaliser et à mettre à disposition des experts associés au processus « formulation recette » sont par exemple :

- Les données fournisseurs (caractéristiques nutritionnelles de chaque matière première et ingrédient). Ces caractéristiques sont reprises manuellement par le chef de projet à partir des fiches techniques de chaque matière première envoyée par les fournisseurs.

- Les estimations faites par le chef de projet R\&D (quantité de chaque matière première et ingrédient mise en œuvre dans la recette $=$ formule recette, humidité finale du produit). 
- Les données clients (poids du biscuit, ingrédients autorisés et non autorisés, exigences nutritionnelles (seuil pour certains nutriments)).

- La formulation de la recette par le chef de projet R\&D qui permet de construire la fiche produit de la recette contenant, la liste des ingrédients, la liste des allergènes (contenus dans la recette), le tableau nutritionnel, les caractéristiques organoleptiques...

Ce travail de formalisation et de mise en perspective avec les experts, de leur rôle et place dans le système, de leurs connaissances, de leurs compétences et de leur savoir-faire, n'avait jamais réellement été mené au sein du groupe. L'organisation de type "libérée » étant fortement fondée sur la responsabilisation et l'autonomie des collaborateurs, et donc sur la prédominance de l'humain dans le système, l'autorégulation, basée quasiment uniquement sur les relations humaines, suffisait, jusque-là, à la conduite du système. L'arrivée d'un PLM bouleverse alors le système sur plusieurs aspects. D'une part, les processus étant mieux définis, les rôles et responsabilités apparaissent plus clairement aux yeux de tous, ce qui est un peu perturbant pour certains : des collaborateurs découvrent qu'ils faisaient, jusque-là, des activités qu'ils ne devaient normalement pas faire ou, au contraire, qu'ils auraient dû en faire certaines. L'autorégulation a conduit à des ajustements internes qui ne nuisent pas forcément au fonctionnement de l'entreprise mais qui trouble un peu la responsabilité. D'autre part, les collaborateurs n'auront, pour beaucoup, plus l'opportunité de mener leurs activités quotidiennes comme ils l'entendent, dans le sens où elles seront, partiellement ou totalement, tracées, et où ils ne pourront, par exemple, plus utiliser les outils qu'ils souhaitent pour gérer et manipuler leurs données. Enfin, il est à noter que classiquement, et peut être plus dans les entreprises "libérées ", la connaissance est un enjeu de pouvoir, et la mise à plat des processus et des données, dans le cadre de ce travail, a conduit à délimiter de nouvelles zones d'influences, ce qui n'est pas non plus évident à vivre pour les collaborateurs, certains se sentant lésés.

\section{Bibliographie}

[BAC 12a] BACZKOWSKI M., « Amélioration du processus de déploiement d'une solution PLM par l'utilisation de cartes heuristiques et de persona : cas LASCOM », Thèse de l’Université Bordeaux 1, soutenue le 12 décembre 2012.

[BAC 12b] BACZKOWSKI M., ROBIN V., Rose B., «Using of the concepts of roles and context in a project management / PLM Solution: The real case study of LASCOM », $11^{\text {th }}$ Biennial Conference on Engineering Systems Design and Analysis (ASME-ESDA2012), Nantes, France, 2012.

[BES 11] BESSON P., Rowe F., «Perspectives sur le phénomène de la transformation organisationnelle », Systèmes d'Information et Management, Vol. 16, $\mathrm{n}^{\circ} 1$, pp. 1-23, 2011.

[BRU 15] BRUNO G., ANTONELli D., Villa A., «A reference ontology to support product lifecycle management », $9^{\text {th }}$ CIRP Conference on Intelligent Computation in Manufacturing Engineering, Procedia CIRP Vol. 33, pp. 41-46, 2015.

[CAN 12] Cantamessa M., Montagna F., Neirotti P., « An empirical analysis of the PLM implementation effects in the aerospace industry », Computers in Industry, Vol. 63, n³, pp. 243-251, 2012.

[CAV 15] CAVAREC Y., « Huit questions sur 1'entreprise libérée », Les Echos, 09/04/2015.

[CHE 09] CHEVAlLEREAU B., « Améliorer les performances de l'industrie logicielle par une meilleure compréhension des besoins », Thèse de l'Université de Nantes, soutenue le 8 Juin 2009.

[DAH 13] Dahmani S., Boucher X., Besombes E., Peillon S., « La trajectoire décisionnelle de servicisation Proposition d'un cadre de modélisation décisionnelle », Conférence Internationale de Génie Industriel, La Rochelle, France, 2013.

[EKA 08] El Kadiri S., Pernelle P., Delattre M., Bouras A., «Pilotage des processus collaboratifs dans les systèmes PLM : Quels indicateurs pour quelle évaluation des performances », $1^{\text {ier }}$ Congrès des innovations mécaniques, Sousse, Tunisie, 2008.

[EKA 09] El Kadiri S., Pernelle P., Delattre M., Bouras A., « Current situation of PLM systems in SME/SMI: Survey's results and analysis », $6^{\text {th }}$ International Conference on Product Lifecycle Management, Bath, UK, 2009. 
[GAV 11] GAVILLET S., «Bien formaliser son projet de GED : préconisations selon le triptyque organisation, technique, humain : Illustration avec le cas du Département Monétique de la Société Général », Mémoire de l'Institut national des sciences et techniques de la documentation, 2011.

[GIR 04] GIRARD Ph., DoumeINGTS G., « Modelling of the engineering design system to improve performance », International Journal of Computers \& Industrial Engineering, Vol. 46, n¹, pp.43-67, 2004.

[GIR 06] GIRARD Ph., RoBIN V., "Analysis of collaboration for project design management », Computers in Industry, Vol. 57, n8-9, pp. 817-826, 2006.

[HeR 05] Hermosillo Worley J., Chatha K.A., Weston R.H., Aguirre O., Grabot B. «Implementation and optimisation of ERP systems: A better integration of processes, roles, knowledge and user competencies », Computers in Industry, Vol. 56, nº, pp. 620-638, 2005.

[KUN 15] KUNG K-H., Ho C-F., HUNG W-H., WU C-C., « Organizational adaptation for using PLM systems: Group dynamism and management involvement», Industrial Marketing Management, Vol. 44, pp. 83-97, 2015. http://www.lascom.fr/marches/logiciel-plm-agroalimentaire/

[LAS 18] LASCOM, Site internet de l'entreprise : http://www.lascom.fr/marches/logiciel-plm-agroalimentaire/, consutlé le $1^{\text {ier }}$ juin 2018.

[LEL 11] LELIEVRE A., «Définition d'un cadre conceptuel et méthodologique pour concevoir un système à flexibilité souhaitée », Thèse de Doctorat de l'Ecole Centrale Paris, soutenue le 12 juillet 2011.

[PET 12] PETERS S., « Et si on partageait le pouvoir dans l'entreprise ? », La Tribune, 22/10/2012.

[PET 08] PETTER S., «Managing user expectations on software projects: Lessons from the trenches », International Journal of Project Management, Vol. 26, nº , pp. 700-712, 2008.

[PIN 17] PinNa C., Plo L., Robin V., GiRARD P., TeRZI S., « An approach to improve implementation of PLM solution in food industry - case study of Poult Group », International Journal of Product Lifecycle Management (IJPLM), Vol.10, n², pp. 151-170, 2017.

[ROB 93] ROBOAM M., « La méthode GRAI : principes, outils, démarche et pratique », TEKNEA, 1993.

[ROS 07] Rose J., Pedersen K., Hosbond JH., Kraemmergaard P., « Management competences, not tools and techniques: a grounded examination of software project management at WM-Data », Information and Software Technology, Vol. 49, n6, pp. 605-624, 2007.

[STA 15] Stark J., «Product Lifecycle Management: 21st Century Paradigm for Product Realization », Decision Engineering, Vol. 1, pp. 235-267, 2015.

[TOP 10] TOPLICEANU G.D., " Prise en compte de l'influence de la culture organisationnelle pour la conduite des activités de conception collaboratives », Thèse de Doctorat de l'Université Bordeaux 1, Janvier 2010.

[WAN 12] WANAVERBECQ C., «L'innovation se niche aussi dans l'organisation », Les Echos, 14/11/2012.

[ZAG 07] ZaghbiB A., Michel C., Prevot P., «Organisation distribuée, mondialisation et R\&D : l'accompagnement des changements organisationnels par des EIAH », $7^{\text {ième }}$ Congrès International de Génie Industriel, Trois-Rivières, Québec, 2007. 